\title{
Multiple Organ Cystic Lesions: A Possible Imaging Target Sign in Von Hippel Lindau Disease - Case Report
}

\author{
Adrian Dumitru Dijmărescu ${ }^{1,2}$, loana Gabriela Lupescu ${ }^{1,2}$
}

Corresponding author:

Adrian Dumitru Dijmărescu, MD

Department of Radiology

Medical Imaging and Interventional

Radiology, Fundeni Clinical Institute

258, Fundeni Street, district 2, 022328

Bucharest, Romania

E-mail: dijmarescu_adrian@yahoo.com

Professor loana Lupescu Department of Radiology Medical Imaging and Interventional Radiology, Fundeni Clinical Institute 258, Fundeni Street, district 2, 022328 Bucharest, Romania

E-mail: ilupescu@gmail.com

\section{Abbreviations:}

VHLD - Von Hippel Lindau disease; US - ultrasound;

CT - computed tomography;

MRI - magnetic resonance imaging; multiple intensity projection (MIP); fat saturation (FS);

Magnetic resonance cholangiopancreatography (MRCP;

diffusion weighted imaging (DWI); apparent diffusion coefficient (ADC); gradient echo (GRE)
'Radiology and Medical Imaging Department, Fundeni Clinical Institute

${ }^{2 "}$ Carol Davila" University of Medicine and Pharmacy, Bucharest, Romania

\section{ABSTRACT}

Introduction: Von Hippel Lindau disease (VHLD) is a rare autosomal-dominant disorder. Multiorgan cysts and low histological grade tumors with various localizations are characteristic for VHLD. Imaging studies are important for the evaluation of VHLD patients.

Case report: We report the case of a 53 years old woman who was admitted for weight loss, chronic abdominal pain and mild jaundice. The patient was known with a cerebral tumor previously removed. Family history was suggestive for VHLD. US showed voluminous cystic lesions developed on the kidneys and in the interhepatopancreatic region with dilated intrahepatic bile ducts. Subsequently contrast enhanced CT and MRI with MRCP were performed. CT showed disorganized pancreatic parenchyma, inhomogeneous by the presence of numerous hypodense lesions, most of them with fluid content, thin nonenhancing walls and small intramural calcifications. Both kidneys had irregular contour, with multiple bilateral cortical cysts and a heterogeneous fast enhancing arterial lesion in the left kidney suggestive for a malignant tumor. MRI showed the same aspect as CT, but also that the lesions exerted a mass effect on the pancreatic tissue and on the Wirsung duct, with no visible communications.

Conclusions: Imaging aspects plays a key role together with the family history for establishing or at least suspecting the diagnosis of VHLD, even though genetic testing is not highly available in all regions. A multidisciplinary team approach is important in the diagnostic and management of VHLD.

Key words: Von Hippel Lindau Disease, pancreatic cysts, renal cysts, solid renal tumor, magnetic resonance imaging, computed tomography

\section{INTRODUCTION}

Von Hippel Lindau disease (VHLD) is a rare autosomal-dominant disorder with a prevalence of 1 in 35000, caused by a mutation in the VHL tumor suppressor gene located on chromosome 3p25-26, which causes VHL protein to be nonfunctional. In most cases the disease is inherited from a parent (more than $80 \%$ ), but de novo mutations are also possible. The VHL gene is involved in the regulation of angiogenesis and cell division. Based on the organ involved and type of tumor identified, different mutations may be determined. Sequencing multigene panels 
are now available, but when there is a high suspicion of VHL mutation, single gene testing may be performed. Multi-organ cysts and low histological grade tumors with various localizations are characteristic for VHLD (1-8).

Imaging studies such as ultrasound (US), computed tomography (CT), and magnetic resonance imaging (MRI) are important for the evaluation of VHLD patients. Hemangioblastomas are often identified on brain and spinal cord magnetic resonance imaging (MRI) $-80 \%$ in the brain vs $20 \%$ in the spinal cord, renal cysts - usually bilaterally, multiple - are seen on ultrasound, CT or MRI, pheochromocytomas on CT or MRI and pancreatic cysts are visualized on US $(4,5,9)$. The pancreas is the site of both cystic and tumoral lesions in patients with VHLD, lesions which may not only be benign (such as cysts, serous cystic adenomas), but also potentially malignant (neuroendocrine) or malignant (metastases) (10-11).

In order to set the diagnosis of VHLD, the following criteria should be met:

- presence of one or more hemangioblastoma of the central nervous system;

- one hemangioblastoma of the central nervous system together with a visceral tumor known to be associated with VHLD;

- any known expression of VHLD when there is a known family history of the disease (12).

\section{CASE PRESENTATION}

We present the case of a 53 years old woman who was admitted to the Gastroenterology Department for weight loss, chronic abdominal pain and mild jaundice. Family history revealed that the patient's father was known with multiple pancreatic and renal tumors. The patient was known with a cerebral tumor which was surgically removed 7 year prior. The current US showed voluminous cystic lesions developed in the interhepatopancreatic region with slightly dilated intrahepatic bile ducts. Similar transonic masses were seen on the kidneys. On US, the lesions were suggestive for simple cysts.

Further imaging studies included CT and MRI.

The CT protocol that we used comprised four phases:

1. a nonenhanced CT phase in order to see changes in the shape, contours, dimensions and structure of the pancreas (atrophy, hypotrophy, fatty infiltration, dilation of the Wirsung duct, calcifications) and most important to provide us the possibility of detecting intracystic densities;

2. three post non-ionic iodinated i.v. contrast CT phases:
- an early arterial phase (20-25 seconds after the start of the i.v. injection) in order to evaluate hypervascularized lesions, a possible vascular involvement and to assess the permeability of the arterial vascularization of the pancreas;

- a pancreatic phase (40-45 sec post i.v. injection) in order to evaluate the enhancement of the pancreatic tissue and the permeability of the spleno-mesenteric-portal venous tract;

- a late venous phase (70-80 seconds post i.v. injection) in order to evaluate the adjacent organs and the entire abdominal venous system.

Afterwards acquisition performed multiplanar (MPR) and multiple intensity projection (MIP) reconstructions were made in order to detect possible involvement of vascular rami.

CT showed that the pancreatic parenchyma was disorganized, inhomogeneous by the presence of numerous hypodense lesions, most of them with fluid content, thin nonenhancing walls and small intramural calcifications. The lesions had a cystic appearance and their dimensions ranged from a few millimeters to 13 centimeters.

In the cephalic and corporeal region of the pancreas voluminous cystic lesions were seen, lesions which were exceeding the margins of the pancreas, with mass effect on the adjacent organs (duodenum, stomach, visceral surface of the liver) and compression on the main bile duct in the hepatic hilum causing slightly dilation of the intrahepatic bile ducts. The main abdominal vessels such as the celiac trunk and its rami and the spleno-portal trunk were also compressed (fig. 1).

The tail of the pancreas presented a few pseudonodular enhancing lesions separated by small layers of pancreatic normal tissue. Malignancy couldn't be excluded at the time.

Both kidneys had irregular contour, with multiple bilateral cortical cysts (Bosniak I), and a heterogeneous fast enhancing arterial lesion in the left kidney with "wash-out" in comparison to the adjacent parenchyma during the following phases (fig. 2), suggestive for a malignant tumor.

The MRI protocol we used was comprised of:

1. $T 1$ and $T 2$ weighted sequences with and without fat saturation (FS), in axial and coronal planes.

2. T2 sequences with long and short TE in axial, coronal and oblique plane.

3. Magnetic resonance cholangiopancreatography (MRCP) acquisition - in order to see if the pancreatic cystic lesions communicated with the 

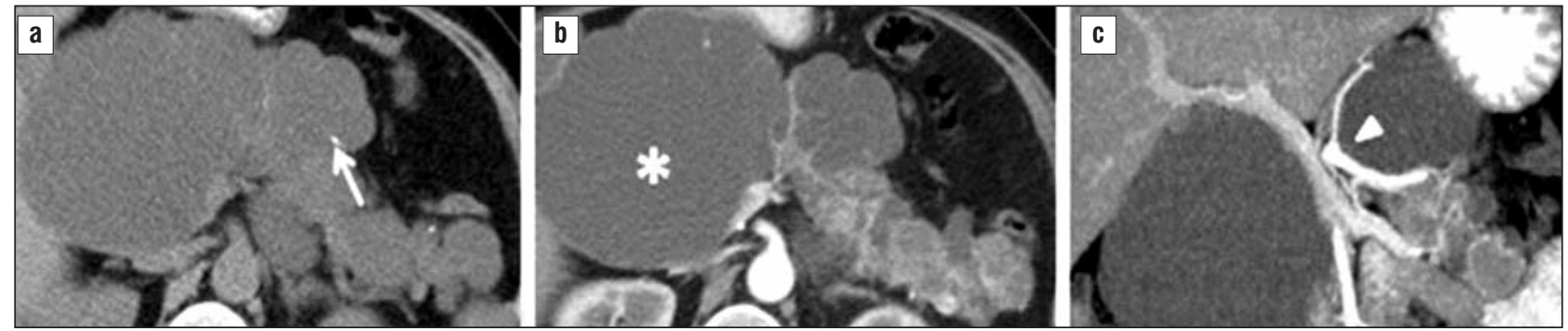

Figure 1 - Pancreatic tissue replaced by numerous hypodense lesions, most of them with fluid content, thin nonenhancing walls and small intramural calcifications (white arrow). Voluminous cystic lesions in the cephalic-corporeal region, exceeding the margins of the pancreas, with mass effect on the adjacent organs (asterix) and vessels (arrow head) - a) nonenhanced CT; b) contrast enhanced CT in arterial phase, c) coronal arterial MIP
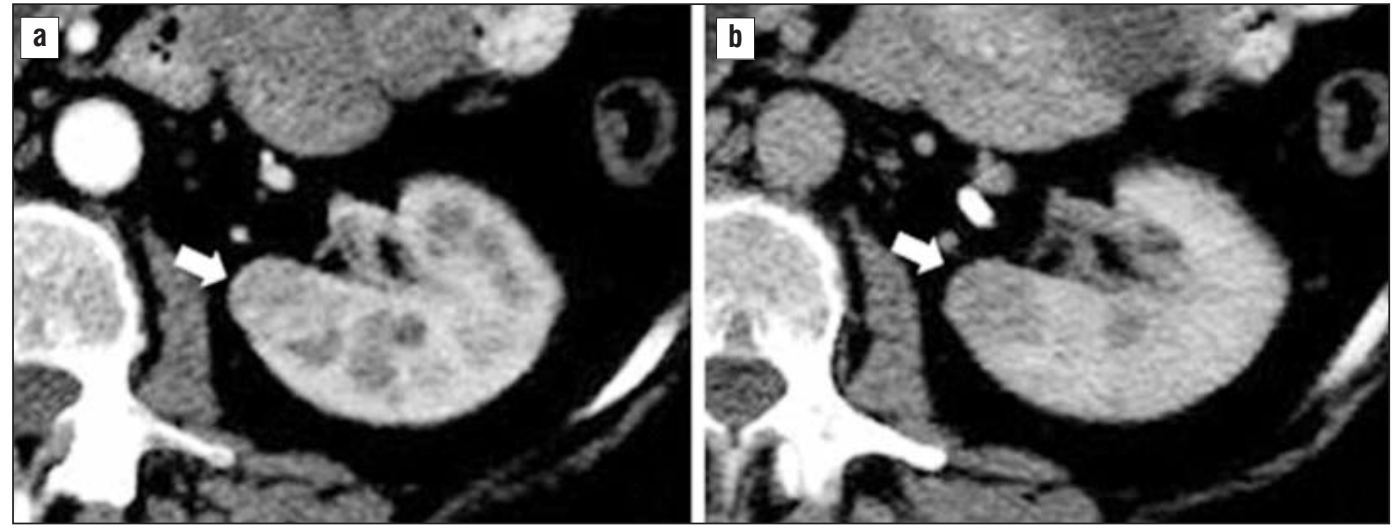

Figure 2 - Heterogeneous (solid and cystic content) nodule in the left kidney with fast enhancing in arterial phase of its solid portions and „wash-out” in comparison to the adjacent parenchyma during the following phases (white arrow) - a) enhanced CT in arterial phase, b) in nephrographic phase

Wirsung duct, the involvement of the main bile duct and also the degree of dilation of the intrahepatic bile ducts

4. diffusion weighted imaging (DWI) sequences with „b" values from 0 to 800 , with apparent diffusion coefficient (ADC) mapping - to delineate between benign and malignant lesions

5. 3DT1 dynamic multiphase acquisition before and after gadolinium i.v. injection

6. T1 in-phase/out-of-phase gradient echo (GRE) sequences - to affirm fatty lesions content or fatty infiltration

MRI showed the multiple cysts previously described in the pancreas by $\mathrm{CT}$, some of them multiloculated with thin septae (,honey comb" aspect). Most of the lesions presented fluid signal, but the largest ones in the cephalic and cephalo-istmic region of the pancreas presented hyperintense T1 signal, with a slightly drop of signal in T2 weighted images, suggesting a modified content (hyperproteic or hemorrhagic), delimitated by thin late enhancing walls, with no focal thickenings, and without restriction of diffusion wi. The mentioned lesions exerted a mass effect on the pancreatic tissue and on the Wirsung duct (evident only in the cephalocorporeal region, with no visible communications) (fig. 3).

In the left kidney the previously described CT nodule was seen - with heterogeneous structure, hypointense in $\mathrm{T} 1 \mathrm{wi}$, hyperintense in $\mathrm{T} 2 \mathrm{wi}$, inhomogeneous gadolinium enhancing pattern, with diffusion restriction and hypoADC appearance - suggesting a tumoral process (renal cell carcinoma). We found the renal cysts with no particular changes except a small one in the left lower pole with modified content (hyperintense T1) (fig. 4).

\section{DISCUSSIONS}

Clinical presentation is variable and nonspecific and the diagnosis is usually established when the patient has VHLD-associated tumors - hemangioblastomas, pheochromocytomas or clear cell renal cell carcinomas and a positive family history for the disease (13). At least 40 different types of lesions assosciated with VHLD 

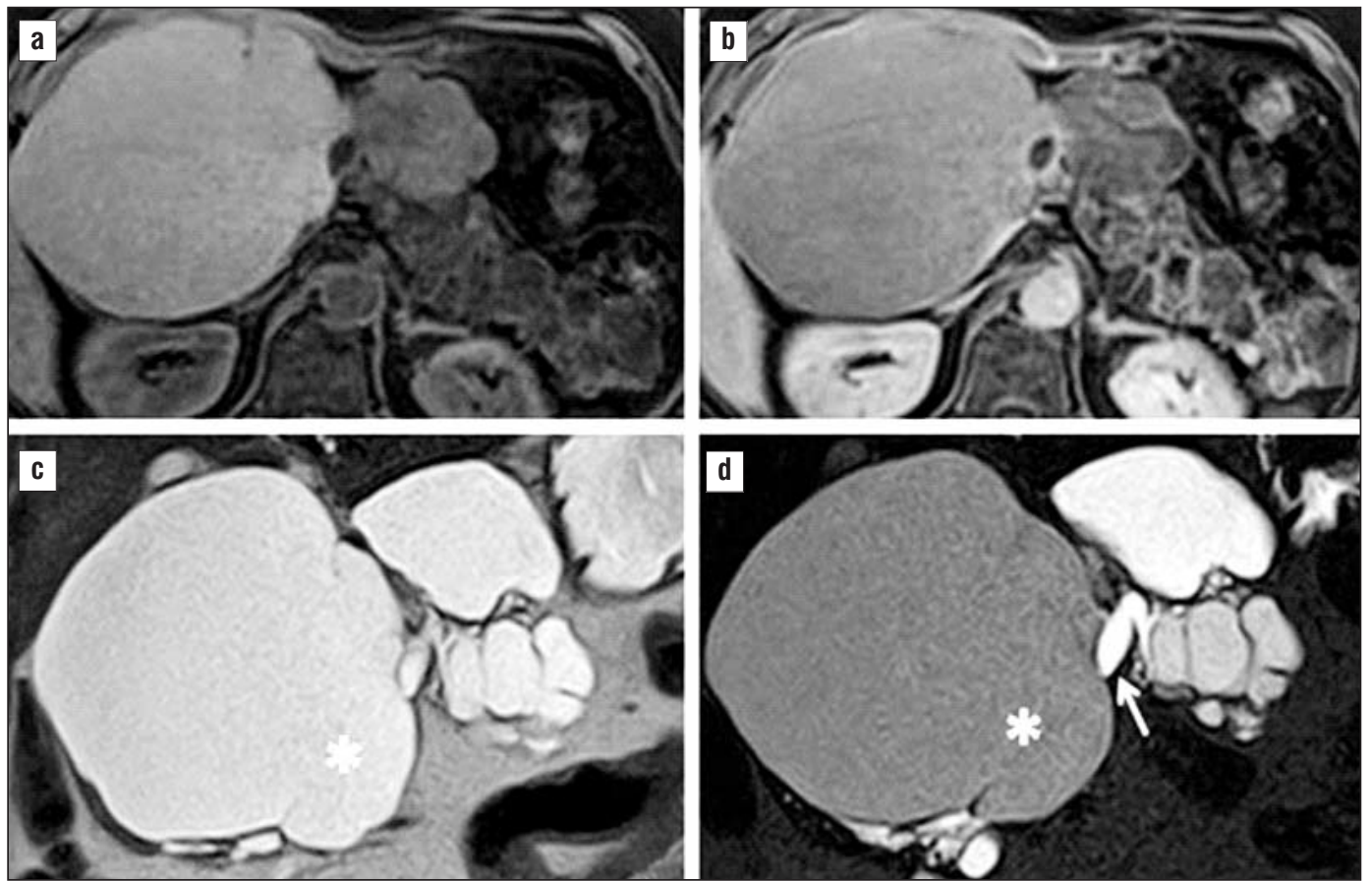

Figure 3 - Multiloculated cysts with thin septae, most of the lesions with fluid signal, but the largest ones located in the cephalic and cephalo-istmic with T1 hyperintensity, with a slightly drop of signal in T2 wi (asterix), with mass effect on the Wirsung duct (arrow), and no visible communications - a) precontrast 3DT1, b) contrast enhanced 3DT1, c) T2 with short TE, d) T2 with long TE.

have been described (12). Most patients have positive family history due to the autosomal dominant inheritance $(9,13)$. The patient presented in this paper was being evaluated for weight loss, chronic abdominal pain and mild jaundice, probably secondary to the large abdominal mass, which was eventually detected by US. Similar to the literature data, a positive family history was identified in this case - the patient's father was
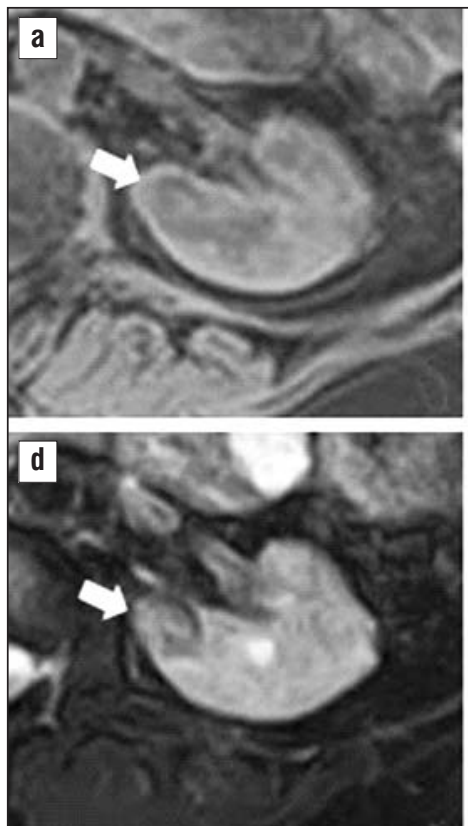
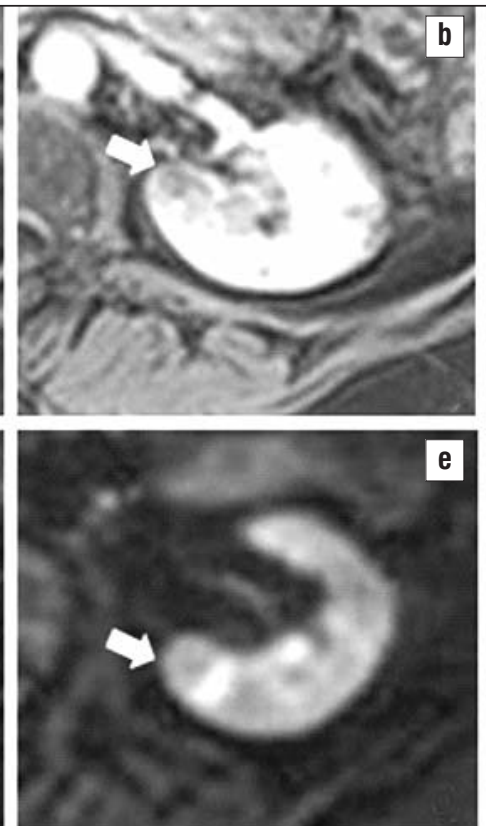
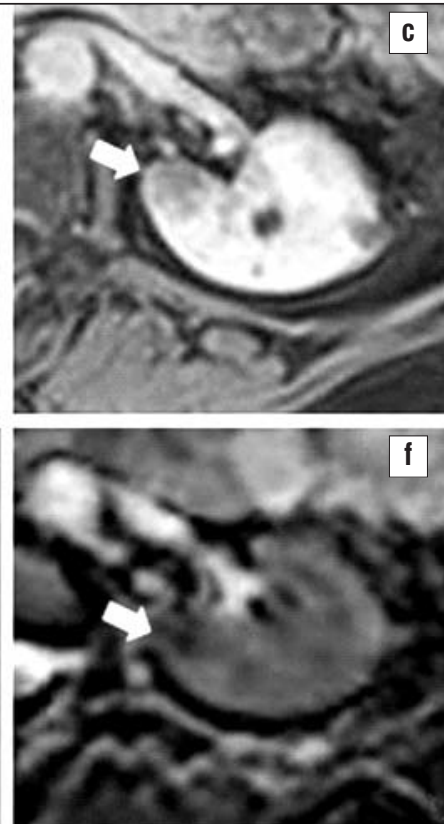

Figure 4 - Heterogeneous renal lesion with restriction of diffusion, hypoADC, low T1 FS signal with heterogenous wash-in/wash-out enhancement (arrow) - a) T1 FS, b)T1+Gd early arterial phase c) T1+Gd late phase, d) T2 FS, e)DWI, f)ADC 
known with multiple pancreatic and renal tumors, but the details of the diagnosis were precarious because genetic testing was not available, not even the histopathologic report. Moreover, the personal history was evocative for VHLD - the patient was known with a cerebral tumor which was removed prior to this episode, but the data regarding the tumor was scarce in this case as well.

The CT and MRI studies showed that the patient had multiple organ -pancreatic and renal- involvement with cysts, some of them simple cysts, others with modified content, and the renal lesion with malignant characteristics - imaging aspect suggestive for renal cell carcinoma.

Considering the imaging aspect previously described and the family history, the most probable diagnosis at this point would be VHLD. Differential diagnosis includes pancreatic cystic lesions and renal polycystic disease for pancreatic and renal cysts and Bourneville syndrome for which renal cysts represent minor diagnosis criteria.

Previous studies report pancreatic involvement in $65-77 \%$ of patients with VHLD, in some of them pancreatic lesions being the only finding (7.6\%). Multiple true cysts appear in up to $91 \%$ of the patients with VHLD and pancreatic involvement $(9,14)$, entity we have also seen in our case. Most patients with these lesions are asymptomatic or present vague symptoms such abdominal pain and mild jaundice (14), similar to our patient's presenting reasons. Clear cell renal cell carcinomas are reported in $24-45 \%$ of patients (9).

Considering the previously stated data, even though we do not have histological data from the surgically removed cerebral tumor, the overall aspect of the other lesions leads us to believe it was a hemangioblastoma tumor usually seen in VHLD $(9,13)$.

Unfortunately, the patient was not genetically confirmed for VHLD, but the imaging aspect shows strong evidence for the disease.

Given that genetic testing is not widely available in some regions, Romania included, diagnosis of VHLD is mainly set based on the family history (not always available and accurate) and on identifying characteristic imaging aspects, raising suspicion. In these regions' awareness of physicians towards this disease is of highly importance.

Even in regions with higher genetic testing availability the disease may remain undiagnosed, in spite of wellestablished international diagnostic criteria, because the disease is not always effortlessly recognized. Early diagnosis is vital for genetic counselling and for surveillance, and it is even more important in patients who remain asymptomatic for long periods of time (6). Moreover, imaging is essential for diagnosing and follow-up of patients with VHLD because identified lesions may be treatable (12). In familial cases the diagnosis may be set on a genetic basis even before the onset of symptoms, unlike the isolated cases, in which the diagnosis is delayed (6).

\section{CONCLUSIONS}

Imaging aspects plays a key role together with the family history for establishing or at least suspecting the diagnosis of VHLD, even though genetic testing is not highly available in all regions. A multidisciplinary team approach is important in the diagnostic and management of VHLD.

\section{Conflict of interest}

All author declare that they have no conflict of interest.

\section{REFERENCES}

1. Varshney N, Kebede AA, Owusu-Dapaah H, Lather J, Kaushik M, Bhullar JS. A Review of Von Hippel-Lindau Syndrome. J Kidney Cancer VHL. 2017; 4(3): 20-29.

2. Figlus M, Kaczorowska B, Jaskólski D, Kepczynski Ł. [Von HippelLindau syndrome - a case report]. Pol Merkur Lekarski. 2018; 22(263): 248-252. Polish.

3. Pilié P, Hasanov E, Matin SF, Woodson AHH, Marcott VD, Bird S, et al. Pilot study of dovitinib in patients with von Hippel-Lindau disease. Oncotarget. 2018; 9(34): 23390-23395.

4. Mikhail M, Singh A. StatPearls. [Online]; 2017 [cited 2018 Jun 21]. Available from: https://www.ncbi.nlm.nih.gov/books/NBK459242/.

5. Leung R, Biswas S, Duncan M, Rankin S. Imaging features of von Hippel-Lindau disease. Radiographics. 2008; 28(1): 65-79.

6. Binderup ML, Galanakis M, Budtz-Jørgensen E, Kosteljanetz M, Luise Bisgaard M. Prevalence, birth incidence, and penetrance of von Hippel-Lindau disease (vHL) in Denmark. Eur J Hum Genet. 2017. 25: 301-307.

7. Nielsen SM, Rhodes L, Blanco I, Chung WK, Eng C, Maher ER, et al. Von Hippel-Lindau Disease: Genetics and Role of Genetic Counseling in a Multiple Neoplasia Syndrome. J Clin Oncol. 2016: 34: $2172-2181$

8. Nguyen TH, Pham T, Strickland T, Brewer D, Belirgen M, Al-Rahawan MM. Von Hippel-Lindau with early onset of hemangioblastoma and multiple drop-metastases like spinal lesions: A case report. Medicine (Baltimore). 2018; 97(39): e12477

9. Yaghobi Joybari A, Azadeh P. Von Hippel-Lindau Disease With MultiOrgan Involvement: A Case Report and 8-Year Clinical Course With Follow-Up. Am J Case Rep. 2017; 18: 1220-1224.

10. Koniusz J, Dabkowski K, Buczek K, Gomółka A, Starzynska T. [Gastroenterological manifestations of von Hippel-Lindau disease]. Pol Merkur Lekarski. 2017; 43(254): 53-55. Polish.

11. Koniusz J, Dabkowski K, Buczek K, Gomółka A, Starzynska T. [Gastroenterological manifestations of von Hippel-Lindau disease a case report]. Pol Merkur Lekarski. 2017; 43(254): 66-68. Polish.

12. Leung R, Biswas S, Duncan M, Rankin S. Imaging Features of von Hippel-Lindau Disease. Radiographics. 2008; 28(1): 65-79

13. Bhuyan M, Dutta D, Baishya B, Hussain Z. Cerebellospinal hemangioblastoma with bilateral pheochromocytoma and hepatic cyst: $A$ rare entity. Asian J Neurosurg. 2016; 11(3): 311.

14. Ma W, Moattar H, Musumba C. Multicystic Pancreas on Endoscopic Ultrasound Imaging: Worth Thinking Outside the 'Box' (Pancreas). Gastroenterology. 2018; 154(4): 814-815. 